\title{
Epigenetic Enabled Normal Human Cells, Lead to First Cell's Unique Division System, Driving Tumorigenesis Evolution
}

\author{
Kirsten H. Walen \\ CROMOS, Richmond, USA \\ Email: kwalencromos@gmail.com
}

How to cite this paper: Walen, K.H. (2022) Epigenetic Enabled Normal Human Cells, Lead to First Cells Unique Division System, Driving Tumorigenesis Evolution. Journal of Cancer Therapy, 13, 48-69. https://doi.org/10.4236/jct.2022.131004

Received: November 28, 2021

Accepted: January 22, 2022

Published: January 25, 2022

Copyright (อ 2022 by author(s) and Scientific Research Publishing Inc. This work is licensed under the Creative Commons Attribution International License (CC BY 4.0).

http://creativecommons.org/licenses/by/4.0/

\section{(c) (i) Open Access}

\begin{abstract}
Normal cells must become cancer-enabling before anything else occurs, according to latest literature. The goal in this mini-review is to demonstrate special tetraploidy in the enabling process. This we have shown from genomic damage, DDR (DNA Damage Response) activity with skip of mitosis leading to diploid G2 cells at the G1 border in need of chromatin repair for continued cell cycling to the special tetraploid division system. In several studies specific methylation transferase genes were activated in normal human cells in tissue fields, containing different cell growth stages of the cancerous process. Histology studies, in addition to molecular chemistry for identification of oncogenic mutational change, were a welcome change (see below). In a study on melanoma origin, DDR also showed arrested diploid cells regaining cycling from methylation transferase activity with causation of $2 \mathrm{n}$ melanocytes transforming to $4 \mathrm{n}$ melanoblasts, giving rise to epigenetic tumorigenesis enabled First Cells. Such First Cells were from Barrett's esophagus shown to have inherited the unique division system from $4 \mathrm{n}$ diplochromosomal cells, first described in mouse ascites cancer cells (below). We discovered that the large nucleus prior to chromosomal division turned $90^{\circ}$ relative to the cytoskeleton axis, and divided genome reductive to diploid, First Cells, in a perpendicular orientation to the surrounding normal cells they had originated from. This unique division system was herein shown to occur at metastasis stage, implying activity throughout the cancerous evolution. Another study showed 4-chromatid tetraploidy in development to B-cell lymphoma, and that such cancer cells also proliferated with participation of this unusual division system. Such participation has long been known from Bloom's inherited syndrome with repair chiasmas between the four chromatids, also an in vitro observation by us. Our cytogenetic approach also revealed that they believed mitotic division in cancer cells is wrong because such cell divisions
\end{abstract}


were found to be from an adaptation between amitosis and mitosis, called amitotic-mitosis. Amitosis means division without centrosomes, which has long been known from oral cancer cells, in that MOTCs (microtubule organizing center) were lacking centrioles. This observation calls for re-introduction of karyotype and cell division studies in cancer cell proliferation. It has high probability of contributing novel approaches to cancer control from screening of drugs against the amitotic-mitotic division apparatus.

\section{Keywords}

Mitotic Slippage, DNA Damage, Repair Process, 4-Chromatid Chromosomes, Diplochromosome Tetraploidy, $90^{\circ}-4 \mathrm{n}$ Nucleus Turn, G1-Phase-Diploid Cell Arrest, Time Reduced Cell Cycle, Fitness Increase

\section{Introduction}

From the inception of LIFE [1], life has been combatting environmental-induced damage to the life-giving DNA molecule, which has led to evolution of survival-selected DNA repair mechanisms. These mechanisms (repair enzymes) became continuously improved upon over evolutionary times, securing accurate chromosomal segregations in mitosis and meiosis, and assurance of repair redundancy mechanisms, should one method fail. For example, a primitive way of segregating genomes for archaic unicellular organisms was that the bichromatic chromosomes formed a ring by end-to-end attachments followed by separations of the rings into daughter organisms [2]. This segregation method was in the absence of centrosome spindle apparatus, which almost a century ago was called amitosis in such primitive unicellular organisms. One cancer scientist claimed end-to-end attachment-residues in cancer cells, also observed in normal human cells [3]. We also found that a special tetraploid division system from 4-chromatid diplochromosomes, distributed diploid genomes to daughter cells in the absence of a spindle apparatus from an adaptation between amitosis and mitosis, called amitotic-mitosis [4]. The name diplochromosomes with 4-chromatid chromosomes was coined from an unusual division system of such $4 \mathrm{n}$ cells in mouse ascites cancer (Levan) [5]. In other cancer cells, centrosomes were found without centrioles, the self-replicating organelle for continuity of centrosomes in "mitosis" [6]. The amitotic-mitosis (see below) division system of the tetraploid cells, were observed to divide successively all the way to haploid cells with rather orderly, accurate whole genome distributions [7] [8]. The extraordinary feature of this tetraploid division system was a $90^{\circ}$ orientation-change relative to the cytoskeleton axis. This unique characteristic occurred all the way to the haploid condition. Haploidy was also observed from embryonic rat cells, but "skewness" of divisions was not mentioned [9]. We also observed that the first tetraploid division gave rise to diploid fitness-gained daughter cells, which were called in vitro, First Cells. Most importantly, the perpendicularity of the division system, 
placed the First Cells, movable-free from Cell Contact Inhibition (a recognized tumor suppressor system) in a perpendicular orientation relative to the surrounding normal cells, which is a requirement for all different tissues giving rise to cancer [10]. This freedom for the fitness-gained First Cell was observed by proliferation being in multilayers with millions of cells in one culture flask, far beyond the 5 - 6 million from normal cells at confluency. Fitness-gain was unexpectedly found to be from reduced cell cycle time, from one mitosis to the next (normal $20-22$ hours reduced to $14-16$ hours). This was corroborated from PtK-cells, time measured from one mitosis to the next in cells marked with tritiated-thymidine uptake and visually shown by autoradiography [11].

\section{Does the Tetraploid Model Apply to Cancer Development?}

The cancer, importance, was in knowing how First Cells originated, which was recently thoroughly, therapy, science and patient, described in a book with the title: THE FIRST CELL [12]. This book, urges a paradigm shift from present whole tumor therapy treatment-protocol, to possible therapy prevention of the cancerous start. We see gain of possible vaccine and therapy information from molecular knowledge of the skewed division system. It should be recognized that the very first break-away of a cell from contact inhibition is not a final occurrence, because the growth from the First Cells will also show contact inhibition from cell-to-cell adherence proteins [13] These proteins only gradually decrease (best known is E-cadherin and $\beta$-catenin) between cells, such that at metastasis, the cells are relatively lightly glued to each other. At the present time, it is only the special tetraploid division system that can achieve such cell release, meaning it has probability of being operational throughout the cancerous process. This consideration is valid for all types of cancers in different body tissues. This first hurdle for cancer development is completely ignored in tumorigenesis ideas by other scientists [14]. However, they talk of EMT/MET embryological occurrences in cancer evolution, but these suggestions are not valid from a more recent study involving special tetraploid division system in ovarian cancers at metastasis [15]. Another fact is that most of the other "cancer" ideas are not from normal human cells, but suggested from cancer cell lines. Below is described the occurrence of this special diplochromosome tetraploid division in cancer cell lines, which is being interpreted to be meiotic-like from whole genome reduction to haploidy. In this paragraph and from earlier reports the conclusion is that the division system of diplochromosomes with $90^{\circ}$ changed orientation is the only known (so far) mechanism that can achieve cell freedom from tumor suppressor tissue Contact Inhibition. As such it is valid for therapy consideration

\section{DNA Damage in the Origin of the Diplochromosome Division System}

Noteworthy, is that this special tetraploid division system, became apparent in normal human cells when they were exposed to DNA damaging agents, medium 
deficient for amino acid glutamine or a highly cell-killing RNA virus. Eons back in time the primitive unicellular organisms also responded with doubling of the genome for chromatid repair, long before mitotic and meiotic evolutionary presence. At that time haploid as well as diploid organisms with such doublings, would have to genome reduce back to living, ploidy level. These whole genome reductive divisions were also in a perpendicular orientation relative to the organismal "-cyto-skeleton-" axis. This produced mirror halves of the daughter cell to be. At that time, repair enzymes were highly important evolutionary tasks, which today are observed as for instance, kinases ATM and ATR, and others. This occurrence has led to cancer therapy by “-drugging DNA repair-", which clinically showed positive results [16], and is certainly supported by our observations from induced DNA damage. Interestingly, repair processes showed focal, $\gamma \mathrm{H} 2 \mathrm{AX}$ on chromosomes in pre-cancer cells [17]. But missing is demonstration of these repair foci on 4-chromatid chromosomes. However, visual chiasmata between the four chromatids of single chromosomes are evidential material for DNA repair [11]. To remember, the $90^{\circ}$ nuclear turn will of course, vary from $0^{\circ}$ to $90^{\circ}$, depended on tissue-tightness, and will therefore be addressed of being “-skewed-". Since we also observed diploid proliferating First Cells with a skewed nucleus, we speculated that they could either divide further to (near) haploid cells or return to tetraploid diplochromosomal cells. We found that this latter speculation had merit from an interesting situation in Barrett's esophagus [18]. The authors, cytometric isolated $4 \mathrm{n}$ cells from pre-cancer growth, and put these cells into cell culture medium for some 20 days. Then cytometry harvested the cell increased cell population, which showed only two peaks $2 \mathrm{n}$ and $4 \mathrm{n}$ cells, no aneuploidy. There is only one explanation; the seeded $4 \mathrm{n}$ cells reduced to $2 \mathrm{n}$ cells, and these $2 \mathrm{n}$ cells genome doubled back to $4 \mathrm{n}$ cells. A rather cancer important cycling: $4 n>2 n>4 n>2 n>$ etc., apparent post oncogenic transformation with activated telomerase gene and conse-quent gain of immortality (below).

\section{The Speculated Division to Haploidy}

Cytogenetics also revealed that haploid cells were marked by $1 / 2$ volume reduction relative to the size of diploid cells, which in leukemia cancers was referred to as "small cells" [19] [20]. We observed the in vitro haploid cells, doubling their genome and start proliferation, notably in "-mitosis-" showing small star-like rosette figures, lying side-by-side on the metaphase plate, similar to the larger rosettes from special tetraploid first divisions [21] [22]. The conclusion is that haplodiploid (46 $\pm 1-2-3$ ) cell proliferation is also from amitotic-mitosis, from absence of anaphase segregated distance between the separated genomes. This amitotic-mitotic adapted division system is not completely unknown: Drosophila cultured cells also from nutrition stress, showed $4 \mathrm{n}$ cells reducing to diploid cells, claimed to be from amitosis, and that the diploid "-First Cells-" proliferated to cancer-like, heaped cell masses [23]. An addition is that the hap- 
lodiploid cells retained the small-cell phenotype, an important fact, perhaps in small-cell solid cancers. The basic underlying maternal-paternal (mat-pat) genome segregation [19] [20] to these haplodiploid cells with amitotic-mitosis proliferation, again point to therapy possibility of such feared, resistant tumors. We have also realized that the Barrett-type cycling $(4 n>2 n>4 n$, etc.) is indicated in the 3-D array, karyotype studies of cancer cells [24] [25] [26] [27]. These array studies, showed ploidy level up, reducing to original level, whereas ploidy level down, produced proliferative cells (the $2 \mathrm{n}$ or haplodiploid $2 \mathrm{n}$ cells). These authors however, argued that aneuploidy caused tumorigenesis initiation and also cancer development, a theme supported by another cancer biologist [28], which is contrary to oncogenic tumorigenesis being preceded by pre-cancers (e.g., colon adenoma and breast in situ lesions). Such cancers proliferate with normal diploid karyotypes, which do not exclude molecular genomic changes. Supportive to diplochromosome cancer initiation and development is the long known hereditary, recessive Bloom's syndrome, which divide by diplochromosomal cells, caused by a helicase mutation with result of genomic doubling to such 4-chromatid chromosomes [29] [30] [31]. Photographic shown diplochromosomal cells expressed excess chiasmata in recombination repair, with the chiasmata between the four-chromatids of one chromosome, and the number of such chromosomes was 46. Recently there have been several suggestions of the 4-chromatid chromosomes being meiotic tetrads from homologous chromosome paring, which implies that we should have seen 23 diplochromosomes, but we did not [32]. Recently, all these unique diplochromosomal division behaviors, were discussed from likely, and proven, cancer occurrence, in regard to in vivo occurrence of First Cells from normal human cells [33].

\section{Another Popular First Cell Proposal}

This proposal is based on an detailed article, The Life Code [34], which suggests de-differentiation of normal cells to embryological germ cell stage for cancer cell origin, gaining various embryological cell traits, in cancer development, especially in regard to giant cell origin (multinucleated cells). News today is that human egg cell-largeness (Giant annotated, but from nutrition proteins) was proposed to be inherited throughout the embryonic development, and in cancer giant cells, the Polyploid Giant Cancer Cell (PGCC) [35]. Another news in this article was that McClintock's transposon studies [36], somehow "-resets-" the genome, which we do not understand unless it has something to do with the function of these transposons heterochromatin condition? But firstly, an answer to the giant size of PGCC from human egg largeness we observed that ploidy level changes (haploid $>$ diploid $>$ tetraploidy and higher) from normal human cells, was linked to nuclear-size increases [37] [38] [39]. These works also showed that tetraploid cells, underwent fission-division to multi-nucleated cells (MNCs) with budding of diploid cells, but that the proliferative capacity from these budded cells, was very limited (max 3 - 4 doubling), far too short for tu- 
morigenic initiation, which negate giant cells in origin of tumorigenesis. Secondly, back to "reset" of the cancer genome [35], suggested to involve McClintock's studies on transposons, showing variegated position effect (PE). How this enters into the reset of the genome is difficult to comprehend. Perhaps the recent investigation [40] [41] on centric heterochromatin in cancer differentiation from for example, breast tumors with estrogen differentiated phenotype, how such cells could proceed to more differentiated aggressive cancer cells. They found the centric heterochromatin "unfolding" and "bursting", which affected silence or activity of coding genes. This strange behavior is somewhat supported by another "-hot-off the press-" article also on transposon (but from retro-elements) behavior with such genome dissociations and inserting somewhere else in the genome, also affecting transcription PE in development [42]. One specific retro-element-like protein, PEG10 showed binding to its own mRNA, secreted in virus-like capsids, found extracellularly, which could act in amino acid transfer into the cell. Importantly, from the many other mentioned similarly acting proteins, the thought was that agents against these proteins might work in nutrition-type therapy. As mentioned these peculiarities are seldom described from normal human cells, but from cancer cell lines with chromosomal scramble genomes, see karyotypes in [26] [27] [28] [29], hardly capable of normal cell activity. No doubt, knowledge of First Cell origin [12], for therapy control of this deadly disease, is a promise.

\section{Details in the Origin of the Diplochromosome Division System}

In this present mini-review with the goal of finding cancer cell behaviors supportive of our idea that the unusual tetraploid division system is an in vitro model for tumorigenesis, the first question is the origin of its underlying genetic system. A possible answer was found in the division system of an extant archaic, unicellular organism, Aluchanta scolymantha [43] before evolved mitotic or meiotic division systems. Such unicellular organisms would then be depended on "amitosis" (above), which also performed repair of DNA with doubling of the genome for recombination repair [44]. The division system segregated whole genomes, which were in a perpendicular orientation to the axis of the organism, achieving mirror halves for daughter cell development. And, in repair of DNA, the doubled genome had to reduce back to living ploidy levels. Note, haploid organisms also with repair of DNA, would have to reduce the diploid recombination genome back to haploidy [44]. We have published about hundred division figures from the special diplochromosomal cells, showing whole genome separation, and the tetraploid nucleus in a $90^{\circ}$ turn relative to the cytoskeleton axis, before the genome reductive division [45] [46]. We conclude that the normal human cells, which responded to DNA damage, possessed evolutionary conserved, ancient unicellular division system. At this time, it is only the diplochromosomal division system that places nuclei and divisions in a $90^{\circ}$ changed 
orientation relative to the cells' cytoskeleton axis. (This whole chapter was intermediately language changed)

In colon hyperplasia with APC mutation, the growth was measured to be in a $90^{\circ}$ orientation-change relative to the basal membrane [47], and tetraploid cells accumulated in this pre-cancerous growth. Here the conclusion is that this in vivo tumorigenesis, originated from DNA damage and the cells responded with DDR activity to special tetraploid division system. This is a second in vivo (Blooms syndrome) occurrence of the in vitro tumorigenesis model. Although the APC-mutation caused microtubule abnormal chromosomal segregations, the surviving cells were karyotype normal. This is because, hyperplasia, in general, is still controlled from normal cell genes. It is only after oncogenic transformation that the genome has become tolerant of aneuploidy, which is shown by divisions being in the tetraploid-triploid level. Ansions were observed to express amitotic-mitosis from "-spindle fibers not coming to a point-" because of no centrioles in the special tetraploid tolerated division [5] [23] [48]. Together with other cancer scientists, the initiation of the cancerous process is concluded to be an atavistic happening from evolutionary conserved primitive division traits in the human genome [49] [50]. In agreement with Vincent, we also see the cancer evolution being from loss of function genetics, figuratively, as a goal in the many years development to the metastasis stage. At this stage the cancer has gained a parasitic life style, by others expressed as a species evolution with autonomy in life style [24] [25]. Therapy at this point, is too late, because the cancer is now sharing life-important genetic-epigenetic systems biology with the host, the patient.

\section{How to Decide Between “(Segregating Diplochromosomes or Synappsed Homologs)"}

This parenthesis quote is from Erenpreisa and Crag [32], supported with more data [51] [52]. Above, it was mentioned that meiosis with homologous chromosome pairing was suggested to explain the four-chromatid condition, being meiotic tetrads [32]. This explanation for the 4-chromatid phenotype of the tetraploid division system, as argued, is not valid because of 46, 4-chromatid chromosomes and not 23 from pairing of homologous chromosomes. The authors based their suggestion on observations of up-regulated meiotic genes in X-rayed cancer cell lines (MOS. REC8, SGO1, SGO2, DMC1, SPO11, SCYP1, STAG3). This important identification suggests X-ray-induced DDR activity with mitotic slippage process to re-replicated genomic cells. We suggest that the genome reductive division of these higher ploidy cells, occurred from activity of the up-regulated genes, genome conserved in the human genome, from primitive unicellular organisms in their fight for survival control of DNA damage. Thus, these genes may be biomarkers for therapy control of the diplochromosomal tetraploid division system. This suggestion is supported by an early study claiming that MOS gene over-expression was induced by Mo-MuSV 124 RNA virus in Swiss 3T3 
mouse cells, (a pro-oncogenic cell line) with result of tetraploid cell-presence from diploid cells [53]. These $4 \mathrm{n}$ cells were photographically shown to have the nucleus in a perpendicular orientation, relative to the longitudinal axis of the cell, which no doubt, was from diplochromosome tetraploid division system. Furthermore, the authors observed bi-nucleated cells, and that the spindle apparatus was an astral (without centrosomes) from altered mitotic spindle poles, attached to the cell membrane "-that interferes with cytokinesis but not karyokinesis-", which is a strong indicator of amitotic-mitosis in these cells (the whole paragraph is intermittent changed).

\section{Gonomery: Maternal Genome Separation from Paternal to Haploid Small-Cell Cancers}

We have published 4 articles on Gonomery; the ability of cells to divide to haploidy from separation of maternal genome from paternal, photographic illustrated in references [9] [18] [19] [54]. This haploid division from diploid cells was discovered for a Copepoda primitive organism, called Gonomery [55]. In mammalian cells the following authors also reported on mat/pat genome separation [8] [56] [57] [58]. To these haploidizations, we add the occurrence of sex determination in Coccids by a diploid male beginning changing to female animals by the male genome destruction and the retained genome doubling to female diploidy [59]. In a more recent article on lung and prostate adenocarcinomas, chemotherapy was associated with change to small-cell cancers with neuroendocrine phenotype (SCLC and SCPC) [60]. The title boldly announced, Cancer Origins, with subtitle, "Similar cancers from different source tissues share molecular mechanisms". This article appears based on an earlier article [61], which found that 5 different genes; p53, RB1, BCL2, myrAKT1, and cMYC, given the name PARCB, which when transduced into normal lung or prostate cells and injected into mice (immunogenic deficient), small-cell neuroendocrine cancers appeared. They then reduced the markers of involved genes, one by one, and found that c-MYC and myrAKT were "indispensable" for cancer development. They did not explain why, what do you think? Do we wonder if myrAKT has anything to do with chromatin methylation change?

Karet and Sage [60], adopted this bio-marker information into their study, and did histology from $\mathrm{H} \& \mathrm{E}$ staining, and also tissue dissociation to single cells, which enabled RNA expression profiles from immunofluorescence techniques. An interesting finding was variations within tissue type, for example, 4, different epithelial cell types in adenocarcinomas (Where they different cancer clones?). But regardless of different analytical approaches, these works did not give the wanted answer; how, adenocarcinomas could change to small-cell cancers. Well, no wonder, neither Park et al. [61] nor these latter authors, used the microscope to look at cells in division and, also if ploidy level changes were present? This negative result is not the only one for waste of scarce research money, and it all is from cancer policy of NO karyotype and division study of cancer cells, be- 
lieved to be adequately replaced by comparative in situ hybridization immunofluorescent methodology for genomic changes. Yes indeed, a powerful method, but it is missing the cancer important fact that the division system is not normal mitosis, but amitotic-mitosis, very probable in divisions to the metastasis stage. This ignorance precludes discovery of novel therapeutic information from this cancer proliferative system. Only one, cancer cell cytogenetic study already mentioned [5], done two decades ago, clearly showed for oral $4 \mathrm{n}$ cancer cells that multipolar divisions were orchestrated from centrosomes without centrioles. And the "empty" MTOCs were constructed from the NuMa protein, which could sprout defective, microtubule spindle apparatus, which clearly demonstration amitotic-mitosis in this cancer cell-division system. Read the Saunders et al.'s article [6], and become convinced that we are "missing the boat" from NO karyotype, and $\mathrm{NO}$ cell divisions studies in cancer development. The assumption of normal mitosis in cancer proliferation is wrong!

\section{Small-Cell Cancer Phenotype Has Been Known for Some Time in Pediatric Leukemia}

It is to be remembered that the small cell lung and prostate cancers (SCLC and SCPC) appeared after that adenocarcinomas had been therapy treated from chemo-drugs [59]. It is known that such drugs can induce DNA damage and DDR activity, which is the route to special tetraploid cells, which now, must be acknowledged to divide all the way to haploid cells [10] [19] [20]. In cell culture from normal human cell strains, this division to haploid cells was associated with genome doubling to near-hypo-diploid cells in proliferation (i.e., haplodiploid). The interesting fact is that the small-cell, $1 / 2$ diploid size reduced, was retained for the haplodiploid cells. Their proliferation were also from amitotic-mitosis from the mentioned fact of small, star-like rosette figures lying side-by-side on the metaphase plate without anaphase distance, similar to the larger rosette from tetraploid division. Two articles are relevant to some degree to these in vitro observations [42] [62]. The latter article, Clipponi et al. discussed the prevalence of genomic damage in cancer development and singled out 10 different chemo-drugs that induced such damage, which was verified by $\gamma_{-}$H2AX repair foci. They concluded: "-DNA damage is a recurrent feature in human cancers exposed to non-genotoxic therapy-". Our translation is: cure-type meant therapy, promotes resistance to therapy and causes relapse cancers through therapy drug-induced genomic damage, which is the route to special tetraploid division system having gone through mitotic slippage process (absence of mitosis). But before leaving this unique division system, another study, already mentioned, from ovarian cancer cells at metastasis [15], showed abnormal, direct divisions to 2,3 , and rarer 4 products, which is a diplochromosome feature [6] [7]. In some pediatric leukemia haploid karyotypes proliferated, and were early described as small cells [57]). Also earlier was small cell lung cancers (SCLC) described from SNP analyzed cells, which showed excessive 
expression of bi-allelic homozygosity, which is indicative of haplo-diploidy [63]. Time has come to investigate the frequent occurrence of uniparental disomy (UPD) in hematological pre- and fully developed mature cancers. Such occurrences can be from haplo-diploidy or a type of so-called partial haploidization, revealed in comparative genome in situ hybridization.

\section{Genome Damage Induced DDR Activity, Needs Studies, Specific for Cell Cycle Effect}

A commentator [64] on Martincorena many works, [65] [66] [67] [68] expressed that genome damage was the base for cancer development, which as mentioned abgove was also the view from Clipponi et al. [42], in spite of all these scientists being firm "believers" in SMGs being initiating and driving tumorigenesis. This pervasive view was also shown in an "all-inclusive" article by Garraway and Lander [69]. Contrary, there are also those that show inactivation of such SMGs [70], which are detected as CNA/Vs (copy number alterations/variabilities), and by HSRs (homogeneously staining regions) and DMs (double minutes) [71]. There is hardly a solid cancer without these latter chromosomal abnormalities. In one photograph diplochromosomes were seen in presence of DMs, but not reacted to for more such observations in the cytogenetic studied ovarian cancer cells. In earlier literature on the evolutionary tree, this loss of function genetics was referred to as an Aneugenic process, which in cancer development was especially demonstrated by Davoli et al. [70]. Another way to loss of function genetics comes from loss of tumor suppressor genes, and reported high frequency of LOH (loss of heterozygosity), which often express haploinsufficiency. Above it was mentioned that this loss of function is a neutral route to autonomous parasitic lifestyle of tumorigenesis [24] [49]. In the end at metastasis stage, the tumor mostly shares genetic/epigenetic traits in common with the patient, which is inhibiting to meaningful therapy approach. This latter situation is hoped to be alleviated by so-called synthetic lethal, which when reaching a threshold in homozygous condition would be cancer deadly. There are also concentrated efforts to discover tumorigenesis as early as possible from liquid biopsies. In this regard, it should also be remembered that metastasis was associated with tetraploid division, which suggests that this skewed division system operated throughout the cancerous process, and therefore should be therapy investigated. The molecular landscape of this special tetraploid division system should be explored for biomarkers targetable from therapy agents. From the further data presented below, the possibility is that such treatments could be the Achilles heel, in the cancer world (in prep.).

\section{1. "The Cell of Origin for Barrett's Esophagus"}

The importance of knowing the First Cell in cancer development [12], described from science, therapy and patient responses ought to be read by all cancer investigators. This suggestion is because the in vitro model for First Cell occurrence is 
gaining in vivo reality almost in leaps from old and new literature findings. For example, a phenomenon, almost 2 centuries old, was brought up for cancer investigation, the fact that cancer growth showed metaplasia, Wikipedia defined it as one cell type giving rise to another type [72]. In von Hansemann's time metaplasia meant squamous cells changing to columnar cell proliferation, an $\mathrm{X}$-axis growth giving rise to a $\mathrm{Y}$-axis growth, which was present in tumorigenic [73]. The present authors [72] turned to pre-cancer and adenocarcinoma study in Barrett's esophagus with the conclusion that information on metaplasia origin "-may lead to better diagnosis, stratification, and treatment, (which) might help to develop targeted chemo-preventive strategies in the future-". They did histology studies of the gastroesophageal junction, and of tissues surrounding this region, and looked for clues that could inform on how the change from squamous to columnar cell type could occur. Important, because the columnar cells gave rise to pre-cancer lesions, a similar study [74], also hot-off the press, ended with same type negative results. We see these failures as above from lack of cancer cell proliferate study (histology study is not5 enough), and also from lack of cancer biology teachings in Med schools. Even cancer pathology teaching, is inadequate for a cancer research career, unless the pa6thologist shows interest outside of book learnings for diagnosis and prognosis. It is hoped that the new ARPA-H, $\mathrm{NIH}$ system, will include cancer biology requirement in Med-school teaching, if the student plans a cancer investigative career. To us metaplasia is a result from special tetraploid division system in a $90^{\circ}$ orientation-change relative to the cytoskeleton axis (the longitudinal axis of the cell, 53). This cancer important trait, placed First Cells with cell polarity change in a perpendicular orientation relative to the surrounding normal cells, meaning a metaplasia origin. The cell polarity change was rather well expressed by budded cells from MNCs [38] [39], and is overwhelmingly shown in RNA virus consecutive killings of surviving re-growths, strangely showing a reproducible sequence of cell-shape changes from fibroblastic, to spindle cells to polygonal cells and last to roundness type cells (WGoliat). Most importantly, these cell polarity changed cell growths were indistinguishable from pathology used in cancer diagnostic and prognostic decisions.

The tumor driver phenotype of SMGs needs clarification (new considerations

In cancer therapy, the technological recent developments, especially in protein chemistry and in gene editing, the CRISPR technology [75], are very impressive, and are being used in several different approaches, but are we dealing with the right target(s)? This question is seen in an article on "The Chromatin of Cancer" [76], which claims that the nature of the tumor driving force(s) is unclear, meaning how the SMGs are driving tumorigenesis: We have also voiced this problem, and have shown that about 25 tumor mutations, including several SMGs are chromo-some located on edges of dark G-bands (coding gene-rich), bordering white regions (gene-poor), because of being regions with non-coding repetitive DNAs, 4 types known. When the cell cycle is time-disturbed from repair of DNA in S-period while the cell is replicating, these regions and mitosis take the brunt. The repetitive regions can show under-replicated deficiencies and mitosis gets 
skipped (mitotic slippage process). Deficiency repair response (DDR) is bound to be a trial and error issue, with causes genome instability, and, mutations in the nearby chromosomal coding gene [77]. This activity invalidates the thinking that the SMGs are selected for in tumorigenesis, and therefore, suggested to drive tumorigenesis. According to Glovers' lifetime, scrutiny of these unstable chromosomal regions, the underlying reason was found to be easier breakability of these sites from naturally weak structures, compared to the rest of the genome. This suggests that chromosome break-ability (with repair process) becomes a factor in the individual frequencies of the different SMGs. The highest frequency for $\mathrm{p} 53$ gene, located on the border of centric heterochromatin on chromosome \#17p-arm, was used to explain how this frequency could be dictated from 3 different insults, whereas most derive from one "-repetitive insult-" [59].

\section{Epigenetic Regulation in Cancer Origin}

Presently there is high attention to also another old observation in cancer occurrence, the fact that stages of oncogenic development can be studied in tissue fields from normal cells to the mature cancer phenotype. The focus is on epigenetic methylation changes, which can occur on DNA nucleotides with causation of gene inactivation or on chromatin histone changes, wound around the helix. It is this chromatin methylation change that is receiving a deeper insight from all field tissue changes including, the normal cells. From the several articles from the Martincorena laboratory, commentators and others [78] [79] [80] reported on chromatin remodeling in urothelial and bladder tissue fields, [65] [66] [67] [68]. In these fields, normal cells were also found to be methylation chromatin changed from the same methylation transferase genes expressed in the various oncogenic growths in the field. These methylation changes were from transferases KMT2D (Mll2), KDM6A (UTX), and ARID1A. But confusing to these believers in the tumorigenesis driver mutation theory (MT) was that the normal cells in the fields were positive for these methylation genes. This led one commentator to write, "-transition from healthy tissue to cancer may be more complicated than we have modeled-". [78]. Furthermore, the expression of these different transferases in different patients was also confusing with for example, “-35 distinct KDM6A mutations distributed over multiple clones and 2 different ARID1A-", in one patient and in another "-4 different KDM6A mutations and 20 different ARID1A", multiple clones [64], It led the same commentator to ask,

"-What is the role of mutations in tumorigenesis, and what factors are required to progress to cancer-" [78]? As above, this question coming from MT believers, ought to lead to more information studies of the true tumorigenesis driving force. One article [66], lists 15 different references to similar histone, chromatin methylation changes for normal cells suspected of tumorigenesis initiation. Does this mean that chromatin methylation change in normal cells is a universal requirement for tumorigenesis initiation? 


\section{Epigenetic Cancer Therapy}

The above question is starting to become a realistic situation (see also below), which has not been missed by the cancer therapy world. For example, the following five different drugs have been FDA approved against lymphoma and myelodysplastic syndrome: vorinostat, romidepsin, 5-azacytidine, decitabine, and ruxolitinib [81]. There are promises for more cancer types to be treated when their transferase genes become revealed. Interestingly environmental factors as smoking and alcohol can "-elicit epigenetic changes-" [82]. Our question is whether this "environmental" effect involves DNA damage to special tetraploid division system? Hampering this therapy approach is the fact that several transferase genes become inactivated with no protein for therapy targeting [83]. But apparently use of HeLa T1 cells with some complex manipulations can uncover involved type of transferase gene [84]. There are also discussions on how flavonoids can be used in such therapy [81]. Our discussions herein on methylation chromatin changes in normal as well as in tumorigenic cells ought to boost attention to this type of cancer therapy, valid across tumor types, as said, perhaps the cancer Achilles heel. (See below and highlighted conclusions).

\section{One Article Heading: "-Permissive Chromatin Landscape-", in Origin of Melanoma}

The quoted above article, plus a commentators' view, discuss how normal cells become melanoma "enabling", which of course in this article is considered from oncogene BRAF of being enabling [85]. Whereas the observed melanocyte transformation to melanoblast, goes almost unnoticed. We shall not discuss the chosen BRAF route, but briefly consider the "-permissive chromatin landscape-", in regard to the mentioned blast cell transformation. In other cancers the change to blast cells is a change from diploid to tetraploid cell cycling, rather well described in [86] from karyotype changes and the worsening of patient's prognosis. Human pluripotent stem cells (hPSCs) for the melanocyte lineage, which when "-disturbed-" (how?) produced melanocytes with requirement of chromatin methylation change [86]. This was observed to be affected by epigenetic regulator ATAD2, transferase gene. The authors claimed that "-Melanoma arise only from cells with a permissive chromatin landscape-". They found that ATAD2 was not only present in melanocytes, but also in tetraploid melanoblast cells, which they claimed gave rise to the origin of melanoma (of course emphasized from BRAF oncogene action). We interpret these observations to mean that ATAD2 transferase gene affected methylation "repair" of "-disturbed-" (above) diploid melanocytes arrested at the G1 border with G2-condensed chromatin. Such diploid cells with G2-phase, condensed chromatin, would be in conflict with G1-phase normal expectation, the de-condensing of anaphase structured chromosomes [46]. The chromatin of such arrested cells "needed" repair for continued cell cycling, which was done by ATAD2 transferase gene. 
This repair permitted S-period re-replication of the diploid melanocytes to special tetraploid, blast melanocytes. Blast cells in other systems have been shown to be aneuploid tetraploidy [86] which is a consequence from oncogenic transformation. In this case of melanoma origin, the re-replication to tetraploid melanocytes from normal melacytes is replication to diplochromosomal melanoblasts, which were found to give rise to melanoma cancer cells. In other words, a rather clear example, of how methylation transferase repaired normal cells can give rise to cancer cells, melanoma cells. The details, preceding the tetraploid blast phase were likely not observable in this study. For instance, the initial replication of the G2-phase cells to diplochromosomal tetraploidy, which does not proliferate as such, but genome reduces to diploid First Cells in precancer proliferation. That melanoma cells were observed means that a precancer cell went through oncogenic transformation process, to the tetraploid melanoblast cells and further to melanoma cancer cells [87]. In addition to Bloom's syndrome this melanoma study is a second example of diplochromosomal division system in cancer initiation and in the development. We urge any scientist who disagrees with the rational used in the melanoma clarification (or in other ideas herein), to publicly debate the issue. Public debate for cancer research has been proposed by Heng [28], and was also an Editorial in Science [88]. Thorp, Editor-in-Chief, argues that "-Public debate is good for science-", and that they should be "-transparent and vigorous-". Something has to give, also an issue from cancer scientist Weinberger, who has advocated for a paradigm shift, similarly expressed by a recommended shift to First Cell-origin [89]. The 4 melanoma-story should have much more attention [90]. It does no good that you and you and I, have our special pet theories on how cancer starts and develops, we have to find commonality in this mess and stop chasing rainbows.

\section{Another Example of Cancer Disease from 46, 4-Chromatid Chromosomes}

Much attention should be given to this article "-Loss of the Suv39h histone methyltransferases-", because the authors used a cytogenetics approach with simple microscopy together with molecular analyses for the unraveling of how B-cell lymphoma originated [91]. They started with normal mouse cells (MEFs, mouse embryonic fibroblasts) and mouse 3T3, non-oncogenic, proliferation-extended cells, and found that centromere heterochromatin became methylated in H3K9 (histone 3 and lysine 9) by two transferase genes Suvh1 and Suvh2. These genedeficiencies, recombination produced in normal and the 3T3 cells, were introduced into mice (chimeric mice), in which B-cell cancers were observed months later. For insight into the "cancer risk", the authors cross-breed the mice to achieve the deficiencies in various combinations, which confirmed the risk to cancer development. They replaced in part (repaired?) these deficiencies transferase deficiencies by substituting these "-evolutionary domains with the bacterial LacZ gene(s)-”, which brings up possible cancer risk from insufficient repair 
of DNA damage, leading to genome instability. Unexpected was that the mice that tolerated the LacZ "-repair-" of the 2 genes, in embryological cells and in the tumors at 9 and 15 months (size-reduced animals with poor survival), showed tetraploid cells with 4-chromatid chromosomes, called butterfly chromosomes (photographic illustrated, no different from human cell diplochromosomes). These chromosomes were also observed in the proliferation of the "half-way transformed" 3T3 cells. The question now is what decided the induction of the butterfly chromosomes, supposedly with special genome reductive division system? The authors explained the 4-chromatid structure from non-dividing centromeres, whereas we are suggesting genome instability from presence of insufficiently repaired deletions of Suvh1 \& 2, the future may decide. Nevertheless, the butterfly chromosomes, undeniably demonstrate that the special tetra-ploidy division system was cancer-initiating and causative of the development to B-cell lymphoma. From all of these different studies, one conclusion is that normal cells must become chromatin methylation remodeled before they can initiate tumorigenesis. A second conclusion is that following mitotic slippage, the ontogeny (birth process) of the special diplochromosomal tetra-ploidy, is the culpritl offender, for required chromatin methylation change in normal human cells.

\section{How Will ARPA-H Claiming "Accelerating Biomedical Breakthroughs", Detect Breakthroughs?}

This Science article [92] explains a long-overdue reorganization of the Health Care System. The outgoing chairman, Francis Collin, will be replaced by several chiefs with divided responsebilities in healthcare, but nothing is said on how these breakthroughs will be recognized. Some scientists have used their own money for patient to voluntarily go through drug trials, which has led to FDA recognition. But that is certainly not a general option. It should also be added that the present atmosphere at $\mathrm{NIH}$, is a conviction in tumor driver mutations being essential for personalized therapy control. The hope for open-mindedness for change is NCI, and TOTT (The Oncology Think Tank), a group of 25 members [93], also with a list of hopeful changes in cancer research.

\section{Conclusion}

In this mini-review of experimental findings in the cancer literature, our in vitro traceable model from diplochromosomal tetraploidy gained evidence of occurrence in initiation and in the developmental process to metastasis malignant stage. In other words, One route to cancer is known from the occurrence of diplochromosomes in Bloom' syndrome and in a B-cell lymphoma development. The basic first step was from damage to DNA, which severe-enough, with causation of mitotic slippage process (skip of mitosis) would produce diplochromosomes with $4-8$ or 8 ) chromatids, a DDR (DNA damage response) for repair activity. As for primitive unicellular organisms, this genomic doubling genome 
reduced back to diploidy, which is the important First Cells for tumorigenesis initiation. Their specialty was shown by being born with fitness-gain, and movable freedom from cell-to-cell, contact inhibition, a basic tumor suppressor system in all tissues giving rise to cancer. This initial tumorigenesis resistance must be overcome, but it has little to no attention in the cancer literature, regardless of type of voiced tumor models (mostly deduced from cancer cell lines). The reason for why the special tetraploid cells achieved this tumorigenesis essential first step, was from the inborn trait of the tetraploid nucleus, turning $90^{\circ}$ before reductive division. This crucial feature placed the progeny, First Cells also in a $90^{\circ}$ relative to the surrounding normal cells free to proliferate from destructions of the adhesion proteins in the normal cell-to-cell adhered condition. However, contact inhibition is a continuous problem in the tumorigenesis evolution, because the First Cell will secret such adherence proteins, which is well known from tumorigenesis gradual loss of E-cadherin and $\beta$-catenin, tight junction adherence proteins. Metastasis depends on "a maximum" loss of such proteins and the question is how it occurs in the years-lengthy evolutionary process? Our guess is from cancer evolutionary occurrence of the 4-chromatid tetraploid division system in perpendicular orientation relative to surrounding cells, setting single cells free from the adherence proteins. Therapy has much to gain from molecular knowledge of this division system, and especially from anti-methylation transferase agents. These approaches have potential of being the Achilles heel of cancer.

\section{Conflicts of Interest}

The author declares no conflicts of interest regarding the publication of this paper.

\section{References}

[1] Loewenstein, W.R. (1999) The Touchstone of Life. Oxford University Press, New York.

[2] Cleveland, L.R. (1947) The Origin and Evolution of Meiosis. Science, 105, 287-289. https://doi.org/10.1126/science.105.2724.287

[3] Walen, K.H. (2009) Spindle Apparatus Uncoupling in Endo-Tetraploid Asymmetric Division of Stem and Non-Stem Cells. Cell Cycle, 8, 3234-3237. https://doi.org/10.4161/cc.8.19.9570

[4] Walen, K.H. (2013) Genome Reversion Process of Endopolyploidy Confers Chromosome Instability on the Descendent Diploid Cells. Cell Biology International, 36, 137-145. https://doi.org/10.1042/CBI20110052

[5] Levan, A. and Hauschka, T.S. (1953) Endomitotic Reduplication Mechanisms in Ascites Tumors of the Mouse. Journal of the National Cancer Institute, 14, 1-43.

[6] Saunders, W.S., Shuster, M., Huang, X., Gharaibe, B., Enyenihi, A.H., Petersen, J. and Gollin, S.M. (2000) Chromosomal Instability and Cytoskeleton Defects in Oral Cancer. Proceedings of the National Academy of Sciences of the United States of America, 97, 303-308. https://doi.org/10.1073/pnas.97.1.303

[7] Walen, K.H. (2007) Bipolar Genome Reductional Division of Human Near-Senescent, 
Polyploid Fibroblast Cells. Cancer Genetics, 173, 43-50.

https://doi.org/10.1016/j.cancergencyto.2006.09.013

[8] Walen, K.H. (2013) Origin of Diplochromosomal Polyploidy in Near-Senescent Fibroblast Cultures: Telomeres and Chromosomal Stability (CIN). Cell Biology International, 31, 1447-1455. https://doi.org/10.1016/j.cellbi.2007.06.015

[9] Glass, E. (1957) Das Problem der Genomsonderung in den Mitosen unbehandelter Rattenlebern. Chromosoma, 8, 468-492. https://doi.org/10.1007/BF01259515

[10] Walen, K.H. (2013) Normal Human Cells Acquiring Proliferative Advantage to Hyperplasia-Like Growth-Morphology: Aberrant Progeny Cells Associated with Endopolyploid and Haploid Divisions. Cancer and Clinical Oncology, 2, 19-33.

[11] Walen, K.H. (1965) Spatial Relationships in the Replication of Chromosomal DNA. Genetics, 51, 915-929.

[12] Raza, A. (2019) The First Cell: And the Human Costs of Pursuing Cancer to the Last. Hachette Book Group, New York.

[13] Sonnenschein, C. and Soto, A.M. (2013) The Aging of the 2000 and 2011 Hallmarks of Cancer Reviews: A Critique. Journal of Biosciences, 38, 651-663. https://doi.org/10.1007/s12038-013-9335-6

[14] Walen, K.H. (2018) Genomic Instability in Cancer I: DNA-Repair Triggering Primitive Hereditary 4n-Skewed, Amitotic Division-System, the Culprit in EMT/MET/Metaplasia Cancer Concepts. Journal of Cancer Therapy, 9, 974-997. https://doi.org/10.4236/jct.2018.912081

[15] Rohnalter, V., Roth, K., Finkernagel, F., Adhikary, T., Obert, J., Dorzweiler, K., et al. (2015) A Multi-Stage Process Including, Transient Polyploidization and EMT Precedes the Emergence of Chemo Resistant Ovarian Carcinoma Cells with a Dedifferentiated and Pro-Inflammatory Secretory Phenotype. Oncotarget, 6, 40005-40025. https://doi.org/10.18632/oncotarget.5552

[16] Jackson, S.P. and Helleday, T. (2016) Drugging DNA Repair. Science, 352, 1178-1179. https://doi.org/10.1126/science.aab0958

[17] Gorgoulis, V.G., Vassillou, L.V.F., Karakaldos, P., Zacharatos, P., Kotsinas, A., Liloglou, T., et al. (2005) Activation of the DNA Damage Checkpoint and Genomic Instability in Human Precancerous Lesions. Nature, 434, 907-913. https://doi.org/10.1038/nature03485

[18] Barrett, M.T., Pritchard, D., Palanca-Wessels, C., Anderson, J., Reid, B.J. and Rabinovitch, P.S. (2003) Molecular Phenotype of Spontaneously Arising 4N (G2-Tetraploid) Intermediates of Neoplastic Progression in Barrett's Esophagus. Cancer Research, 63, 4211-4217.

[19] Walen, K.H. (2014) Neoplastic-Like Cell Changes of Normal Fibroblast Cells Associated with Evolutionary Conserved Maternal and Paternal Genomic Autonomous Behavior (Gonomery). Journal of Cancer Therapy, 5, 860-877. https://doi.org/10.4236/jct.2014.59094

[20] Walen, K.H. (2014) Haploidization of Human Diploid Metaphase Cells: Is This Genome Reductive Mechanism Operational in Near-Haploid Leukemia? Journal of Cancer Therapy, 5, 101-114. https://doi.org/10.4236/jct.2014.51013

[21] Walen, K.H. (2010) Mitosis Is Not the Only Distributor of Mutated Cells: Non-Mitotic Endopolyploid Cells Produce Reproductive Genome-Reduced Cells. Cell Biology International, 34, 867-872. https://doi.org/10.1042/CBI20090502

[22] Walen, K.H. (2015) Wound Healing Is a First Response in a Cancerous Pathway: Hyperplasia Developments to $4 \mathrm{n}$ Cell Cycling in Dysplasia Linked to Rb-Inactivation. Journal of Cancer Therapy, 6, 906-916. 
https://doi.org/10.4236/jct.2015.610099

[23] Lucchetta, E.M. and Ohlstein, B. (2017) Amitosis of Polyploid Cells Regenerates Functional Stem Cells in Drosophila Intestine. Cell Stem Cell, 20, 609-620.E6. https://doi.org/10.1016/j.stem.2017.02.012

[24] Bloomfield, M. and Duesberg, P. (2015) Karyotype Alteration Generates the Neoplastic Phenotypes of SV40-Infected Human and Rodent Cells. Molecular Cytogenetics, 8, Article No. 79. https://doi.org/10.1186/s13039-015-0183-y

[25] Bloomfield, M. and Duesberg, P. (2016) Inherent Variability of Cancer-Specific Aneuploidy Generates Metastasis. Molecular Cytogenetics, 9, Article No. 90. https://doi.org/10.1186/s13039-016-0297-x

[26] Duesberg, P., Madrioli, D., McCormack, A. and Nicholson, J.M. (2011) Is Carcinogenesis a Form of Speciation? Cell Cycle, 10, 2100-2114. https://doi.org/10.4161/cc.10.13.16352

[27] Duesberg, P. and McCormack, A. (2013) Immortality of Cancers: A Consequence from Inherent Karyotype Variations and Selections for Autonomy. Cell Cycle, 12, 783-802. https://doi.org/10.4161/cc.23720

[28] Heng, H.H. (2015) Debating Cancer: The Paradoxes in Cancer Research. World Scientific, Singapore.

[29] Therman, E. and Susman, M. (1993) Human Chromosomes-Structure, Behavior, and Effects. 3rd Edition, Springer, New York.

https://doi.org/10.1007/978-1-4684-0529-3

[30] Kuhn, E.M. (1981) A High Incidence of Mitotic Chiasmata in Endoreduplicated Bloom's Syndrome. Human Genetics, 58, 417-421.

https://doi.org/10.1007/BF00282827

[31] Ellis, N.A., et al. (2008) Bloom's Syndrome Workshop Focuses on the Functional Specificities of RecQ Helicases. Mechanisms of Ageing and Development, 129, 681-691. https://doi.org/10.1016/j.mad.2008.09.005

[32] Erenpreisa, J. and Crag, M.S. (2013) Three Steps to Immortality of Cancer Cells: Senescence, Polyploidy and Self-Renewal. Cancer Cell International, 13, Article No. 92. https://doi.org/10.1186/1475-2867-13-92

[33] Walen, K.H. (2021) Cell Cycle Stress in Normal Human Cells: A Route to "First Cells" (with/-without Fitness Gain) and Cancer-Like Cell-Shape Changes. Seminars in Cancer Cell Biology, in Press. https://doi.org/10.1016/j.semcancer.2020.12.023

[34] Liu, J.S. (2019) The "Life Code": A Theory that Unifies the Human Life Cycle and the Origin of Human Tumors. Seminars in Cancer Cell Biology, 60, 380-397. https://doi.org/10.1016/j.semcancer.2019.09.005

[35] Liu, J.S. (2021) Giant Cells: Linking McClintock's Heredity to Early Embryogenesis and Tumor Origin throughout Millennia on Earth. Seminars in Cancer Cell Biology, in Press. https://doi.org/10.1016/j.semcancer.2021.06.007

[36] McClintock, B. (1984) The Significance of Responses of the Genome to Challenge. Science, 226, 792-801. https://doi.org/10.1126/science.15739260

[37] Walen, K.H. (2002) The Origin of Transformed Cells: Studies of Spontaneous and Induced Cell Transformation in Cell Cultures from Marsupials, a Snail and Human Amniocytes. Cancer Genetics, 133, 45-54. https://doi.org/10.1016/S0165-4608(01)00572-6

[38] Walen, K.H. (2004) Spontaneous Cell Transformation: Karyoplasts Derived from Multi-Nucleated Cells Produce New Cell Growth in Senescent, Human Epithelial Cell Cultures. In Vitro Cellular \& Developmental Biology-Animal, 40, 150-158. 
[39] Walen, K.H. (2005) Budded Karyoplasts from Multinucleated Fibroblast Cells Contain Centrosomes and Change Their Morphology to Mitotic Cells. Cell Biology International, 29, 1057-1065. https://doi.org/10.1016/j.cellbi.2005.10.016

[40] Krigerts, J., Salmina, K., Freivalds, T., Zayakin, P., Rumnieks, F., et al. (2021) Differentiating Cancer Cells Reveal Early Large-Scale Genome Regulation by Pericentric Domains. Biophysical Journal, 120, 711-724.

https://doi.org/10.1016/j.bpj.2021.01.002

[41] Erenpreisa, J., Salmina, K., Anatskaya, O. and Cragg, M.S. (2021) Paradoxes of Cancer: Survival on the Brink. Seminars in Cancer Cell Biology, in Press. https://doi.org/10.1016/j.semcancer.2020.12.009

[42] Clipponi, A., Goode, D.L., Bedo, J., McCabe, M.J., Pajic, M., Croucher, D.R., et al. (2020) MTOR Signaling Orchestrates Stress-Induced Mutagenesis, Facilitating Adaptive Evolution in Cancer. Science, 368, 1127-1131.

https://doi.org/10.1126/science.aau8768

[43] Grell, K.G. and Ruthmann, A. (1964) Über die Karyologie des Radiolars Aulachanta scolymantha und Feinstruktur seiner Chromosomen. Chromosoma, 15, 185-211. https://doi.org/10.1007/BF00285729

[44] Hurst, L.D. and Nurse, P. (1991) A Note on the Evolution of Meiosis. Journal of Theoretical Biology, 150, 561-563. https://doi.org/10.1016/S0022-5193(05)80447-3

[45] Walen, K.H. (2019) Genomic Instability in Cancer II: 4N-Skewed (90) Reductive Division via Fragile Sites to Fitness Increase for Solid and Hematological Cancer Beginnings. Journal of Cancer Therapy, 10, 537-564. https://doi.org/10.4236/jct.2019.107045

[46] Walen, K.H. (2021) A Traceable Cancer Model: DNA Damage, Fragile Site-SMGs, Mitotic Slippage, 4n-Genome-Reduction to Fitness-Gained, Initiating, 2n First Cells. Journal of Cancer Therapy, 12, 365-386. https://doi.org/10.4236/jct.2021.126033

[47] Dikovskaya, D., Schiffmann, D., Newton, IP., Oakley, A., Kroboth K., Sansom O., et al. (2007) Loss of APC Induces Polyploidy as a Result of a Combination of Defects in Mitosis and Apoptosis. Journal of Cell Biology, 176, 183-195. https://doi.org/10.1083/jcb.200610099

[48] Brenner, S., Branch, A., Meredith, S. and Berns, M.W. (1977) The Absence of Centrioles from Spindle Poles of Rat Kangaroo PtK1 Cells Undergoing Meiotic-Like Reduction Division in Vitro. Journal of Cell Biology, 72, 368-379. https://doi.org/10.1083/jcb.72.2.368

[49] Vincent, M.D. (2010) The Animal within: Carcinogenesis and Clonal Evolution of Cancer Cells Are Speciation Events Sensu Stricto. Evolution, 64, 1173-1183. https://doi.org/10.1111/j.1558-5646.2009.00942.x

[50] Niculescu, V. (2018) Carcinogenesis: Recent Insight in Protist Stem Cell Biology Lead to a Better Understanding of Atavistic Mechanisms in Cancer Development. MOJ Tumor Research, 1, 18-29.

[51] Erenpreisa, J., Salmina, K., Huna, A., Kosmacek, E.A., Cragg, M.S., Ianzini, F. and Anisimov, A. (2011) Polyploid Tumor Cells Elicit Paradiploid Progeny through Depolyploidizing Divisions and Regulated Autophagic Degradation. Cell Biology International, 35, 687-695. https://doi.org/10.1042/CBI20100762

[52] Erenpreisa, J., Salmina, K., Huna, A., Jackson, T.R., Vazquez-Martin, A. and Cragg, M.S. (2015) The "Virgin Birth", Polyploidy and the Origin of Cancer. Oncoscience, 2, 3-14. https://doi.org/10.18632/oncoscience.108

[53] Fukasawa, K. and Vande Woude, G.F. (1995) Mos Overexpression in Swiss 3T3 
Cells Induces Meiotic-Like Alterations of the Mitotic Spindle. Proceedings of the National Academy of Sciences of the United States of America, 92, 3430-3434. https://doi.org/10.1073/pnas.92.8.3430

[54] Heim, S. and Mitelman, F. (1995) Cancer Cytogenetics: Chromosomal and Molecular Genetic Aberrations of Tumor Cells. 2nd Edition, Wiley-Liss, Inc., New York.

[55] Rach, E.M. and Wyngaard, G.A. (2008) Gonomery and Chromatin Diminution in Mesocyclops longisetus (Copepoda). Journal of Crustacean Biology, 28, 180-184. https://doi.org/10.1651/07-2847R.1

[56] Glazko, T.T. (2004) Chromosome Subdividing to Haploid Sets in Diploid Metaphase Plates of Some Mammalian Species. Proceedings of the 15th International Chromosome Conference, London, 5-10 September 2004.

[57] Mayer, W., Smith, A., Fundele, R. and Haaf, T. (2000) Spatial Separation of Parental Genomes in Preimplantation Mouse Embryos. Journal of Cell Biology, 148, 629-634. https://doi.org/10.1083/jcb.148.4.629

[58] Haas, O.A. (2021) Somatic Sex: On the Origin of Neoplasms with Chromosome Counts in Uneven Ploidy Ranges. Frontiers in Cell and Developmental Biology, 9, Article ID: 631946. https://doi.org/10.3389/fcell.2021.631946

[59] Walen, K.H. (2020) Near-Dead Cells to Special Tetraploidy to First Cells to Cancer Diagnostic Morphology: Unlikely Therapy-Gain from For-Profit Industrial Goliath. Journal of Cancer Therapy, 11, 410-432. http://www.scirp.org/journal/jctISSNOnline2151-1942 https://doi.org/10.4236/jct.2020.117036

[60] Kareta, M.S. and Sage, J. (2018) Cancer Origins-Genetics Rules the Day. Science, 362, 30-31. https://doi.org/10.1126/science.aav1044

[61] Park, J.W., Lee, J.K., Sheu, K.M., Wang, L., Balanis, N.G., Nguyen, K., Smith, B.A., et al. (2018) Reprogramming Normal Human Epithelial Tissues to a Common, Lethal Neuroendocrine Cancer Lineage. Science, 362, 91-95. https://doi.org/10.1126/science.aat5749

[62] Khan, M.A., Walch, P.C., Miller, M.C., Bales, W.D., Epstein, J.I., Mangold, L.A., et al. (2003) Quantitative Alterations in Nuclear Structure Predict Prostate Carcinoma Distant Metastasis and Death in Men with Biochemical Recurrences after Radical Prostatectomy. Cancer, 98, 2583-2591. https://doi.org/10.1002/cncr.11852

[63] Pfeifer, M., Guesta, L.F., Sos, M.L., George, J., Seidel, D., Kasper, L.H., Plenker, D., Leenders, F., Sun, R., et al. (2012) Integrative Genome Analyses Identify Key Somatic Driver Mutations of Small-Cell Lung Cancer. Nature Genetics, 44, 1104-1110. https://doi.org/10.1038/ng.2396

[64] Rozen, S.G. (2020) Mutational Selection in Normal Urothelium. Science, 370, 34-35. https://doi.org/10.1126/science.abe0955

[65] Nik-Zanail, S., Davies, H., Staff, J., Ramakrishna, M., Glodzik, D., Xou, X., Martincorena, I., et al. (2016) Landscape of Somatic Mutations in 560 Breast Cancer Whole Genome Sequences. Nature, 534, 47-54. https://doi.org/10.1038/nature17676

[66] Martincorena, I., Fowler, J., Wabik, A., Lawson, A.R.J., Abascal, F., Hall, M.W.J., Cagan, A., Murai, K., et al. (2018) Somatic Mutant Clones Colonize the Human Esophagus with Age. Science, 362, 911-917. https://doi.org/10.1126/science.aau3879

[67] Martincorena, I. and Campbell, P.J. (2015) Somatic Mutations in Cancer and Normal Cells. Science, 349, 1483-1489. https://doi.org/10.1126/science.aab4082

[68] Martincorena, I., et al. (2017) Universal Patterns of Selection in Cancer and Somatic Tissues. Cell, 171, 1029-1041.E21. https://doi.org/10.1016/j.cell.2017.09.042 
[69] Garraway, L.A. and Lander, E.S. (2013) Lessons from the Cancer Genome. Cell, 153, 17-37. https://doi.org/10.1016/j.cell.2013.03.002

[70] Davoli, T., Uno, H., Wooten, E.C. and Elledge, S.J. (2017) Tumor Aneuploidy Correlates with Markers of Immune Evasion and with Reduced Response to Immunotherapy. Science, 355, 261. https://doi.org/10.1126/science.aaf8399

[71] Storlazzi, C.T., Lonoce, A., Guastadisegni, M.C., Trombetta, D., et al. (2010) Gene Amplification as Double Minutes or Homogeneously Staining Regions in Solid Tumors: Origin and Structure. Genome Research, 20, 1198-1206. https://doi.org/10.1101/gr.106252.110

[72] Geboes, K. and Hoores, A. (2021) The Cell of Origin for Barrett's Esophagus. Science, 373, 737-738. https://doi.org/10.1126/science.abj9797

[73] Bignold, L.P., Coghlan, B.L.D., Jersmann, H.P.A. and von Hansemann, D. (2007) Contributions to Oncology, Context, Comments, and Translations. Birkhauser Verlag, Basel.

[74] Couzin-Frankel, J. (2020) CRISPR Takes on Cancer. Science, 367, 616. https://doi.org/10.1126/science.367.6478.616

[75] Nowicki-Osuch, K., Shuang, L., Jammula, S., Bleaney, C.W., Mahbubani, K.T., et al. (2021) Molecular Phenotyping Reveals the Identity of Barrett's Esophagus and Its Malignant Transition. Science, 373, 760-767.

https://doi.org/10.1126/science.abd1449

[76] Taipale, J. (2018) The Chromatin of Cancer-Understanding Tumorigenesis and Inherited Risk of Cancer Requires a Multiomic Approach. Science, 362, 401-402. https://doi.org/10.1126/science.aav3494

[77] Durkin, S.G. and Glover, T.W. (2007) Chromosome Fragile Sites. Annual Review of Genetics, 41, 169-192. https://doi.org/10.1146/annurev.genet.41.042007.165900

[78] Chanock, S.J. (2018) The Paradox of Mutations and Cancer. Science, 362, 893-894.

[79] Lawson, A.R.J., Abascal, F., Coorens, T.H.H., Hooks, Y., O’Neill, L., Latimer, C., Raine, K., et al. (2020) Extensive Heterogeneity in Somatic Mutation and Selection in the Human Bladder. Science, 370, 75-82. https://doi.org/10.1126/science.aba8347

[80] Li, R., Du, Y., Chen, Z., Xu, D., Lin, T., Jin, S., Wang, G., et al. (2020) Macroscopic Somatic Clonal Expansion in Morphologically Normal Human Urothelium. Science, 370, 82-89. https://doi.org/10.1126/science.aba7300

[81] Khan, H., Belwal, T., Efferth, T., Farooqi, A.A., et al. (2021) Targeting Epigenetic in Cancer: Therapeutic Potential of Flavonoids. Critical Reviews in Food Science and Nutrition, 61, 1616-1639. https://doi.org/10.1080/10408398.2020.1763910

[82] Wang, T.H., Hsia, S.M., Shih, Y.H. and Shieh, T.M. (2017) Association of Smoking, Alcohol Use, and Betel Qui Chewing. International Journal of Molecular Sciences, 18, Article No. 1210. https://doi.org/10.3390/ijms18061210

[83] Kelly, A.D. and Issa, J.P. (2017) The Promise of Epigenetic Therapy: Reprogramming the Cancer Genome. Current Opinion in Genetics \& Development, 42, 68-77. https://doi.org/10.1016/j.gde.2017.03.015

[84] Maximova, V., Shalginskikh, N., Vlasove, O. and Usalka, O. (2021) HeLa T1 Cell-Based Assay as a New Approach to Screen for Chemicals Able to Reactivate the Expression of Epigenetic Silenced Genes. PLoS ONE, 16, e0252504. https://doi.org/10.1371/journal.pone.0252504

[85] Vredevoogd, D.W. and Peeper, D.S. (2021) Enabling Oncogenes. (Subtitle: Melanoma Can Arise Only from Cells with Permissive Chromatin Landscape) Science, 373, 1088-1089. https://doi.org/10.1126/science.abl4510 
[86] Abe, R., Raza, A., Preisler, H.D., Tebbi, C.K., Sandberg, A.A. (1985) Chromosomes and Causation of Human Cancer and Leukemia. LIV. Near Tetraploidy in Acute Leukemia. Cancer Genetics, 14, 45-59. https://doi.org/10.1016/0165-4608(85)90214-6

[87] Thorp, H.H. (2021) Public Debate Is Good for Science. Science, 371, 213. https://doi.org/10.1126/science.abg4685

[88] Baggiolini, A., Callahan, S.J., Montai, E., Weiss, J.M., Trieu, T., Tagore, M.M., el al. (2021) Developmental Chromatin Programs Determine Oncogenic Competence in Melanoma. Science, 373, 1104. https://doi.org/10.1126/science.abc1048

[89] Walen, K.H. (2017) Mitotic Slippage Process Concealed Cancer-Sought Chromosome Instability Mechanism (S-CIN). Journal of Cancer Therapy, 8, 608-623.

https://doi.org/10.4236/jct.2017.86052

[90] Lindsey, S.F., Byrnes, D.M., Eller, M.S., Rosa, A.M., Dabas, N., et al. (2013) Potential Role of Meiotic Proteins in Melanoma Chromosomal Instability. Journal of Skin Cancer, 2013, Article ID: 190109. https://doi.org/10.1155/2013/190109

[91] Peters, A.H.F.M., O'Carroll, D., Scherthan, H., Mechtler, K., Sauer, S., Schøfer, C., et al. (2001) Loss of the Suv39h Histone Methyltransferases Impairs Mammalian Heterochromatin and Genome Stability. Cell, 107, 323-337. https://doi.org/10.1016/S0092-8674(01)00542-6

[92] Collins, F.S., Schwetz, T.A., Tabak, L.A. and Lander, E.S. (2021) ARPA-H: Accelerating Biomedical Breakthroughs. Science, 373, 165-167. https://doi.org/10.1126/science.abj8547

[93] Raza, A., et al. (2021) We Must Find Ways to Detect Cancer Much Earlier. Scientific American. 